\title{
Evaluación de morfotipos de coral Acropora cervicornis en guarderías colgantes para restauración
}

\section{Evaluation of Acropora cervicornis Morphotypes in Hanging Nurseries for Restoration}

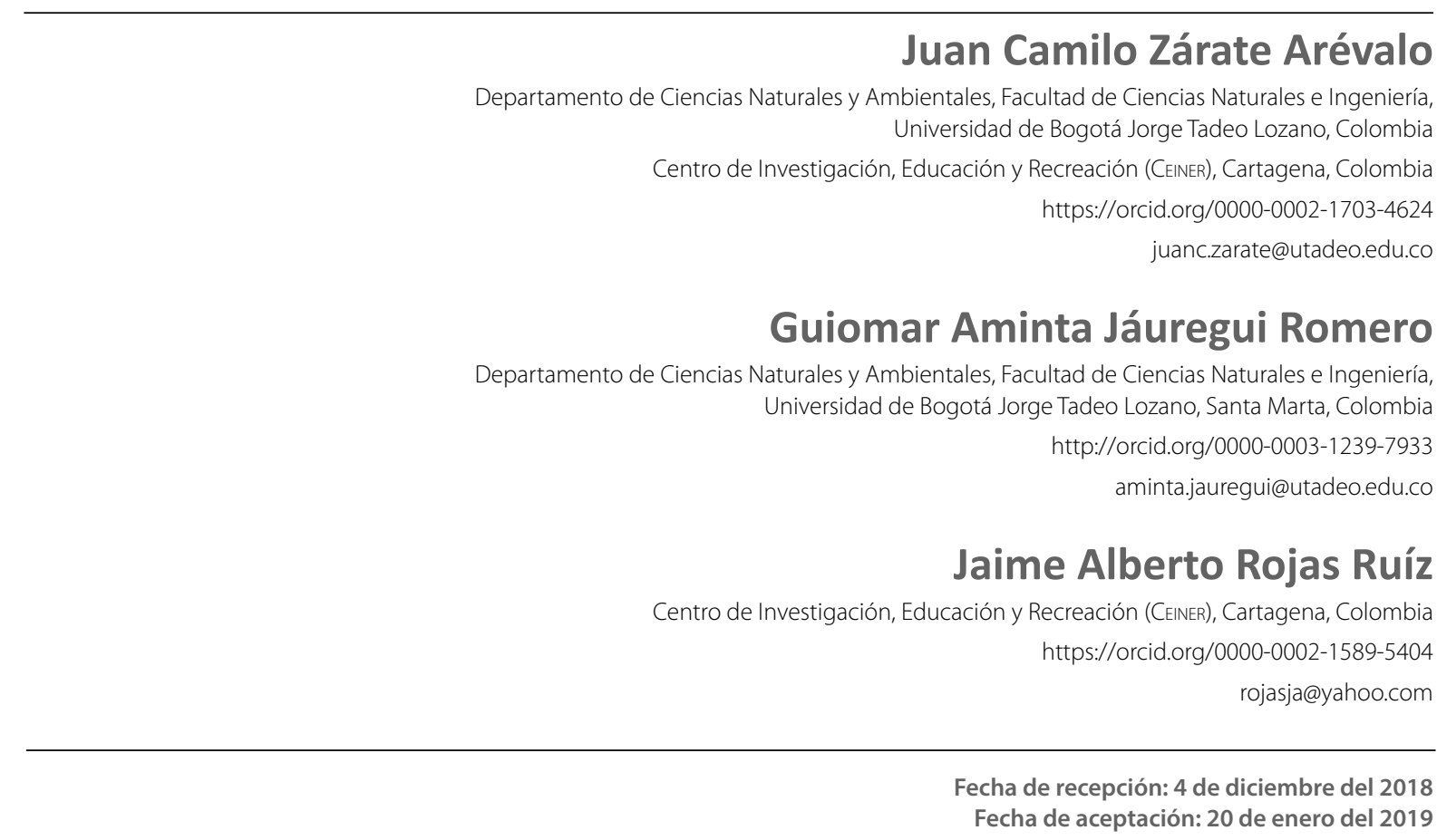

Sugerencia de citación: Zárate, J. C., Jáuregui, A. y Rojas, J. (2019). Evaluación de morfotipos de coral Acropora cervicornis en guarderías colgantes para restauración. Mutis, 9(1), 9-19, doi: 10.21789/22561498.1452

\section{RESUMEN}

La degradación acelerada de las formaciones coralinas, especialmente de las especies de aguas someras como A. cervicornis, ha causado preocupación. Por esta razón, se han implementado diferentes medidas activas para recuperar este ecosistema. El presente estudio evaluó la eficiencia de un modelo de guardería vertical tipo árbol con fragmentos de A. cervicornis en la isla San Martín de Pajarales (Oceanario, Centro de Investigación, Educación y Recreación [CEINER], Parque Nacional Natural Corales del Rosario y San Bernardo [PNNCR y SB], Colombia), donde se instalaron 10 guarderías verticales (5 morfotipos del coral). De cada morfotipo se tuvieron en cuenta 12 fragmentos, para los cuales se determinó su crecimiento (lineal y volumen ecológico), sobrevivencia y estado de salud. Los morfotipos evaluados se comportaron de manera similar en ambos tipos de crecimiento. Los 
resultados de esta investigación señalan que la sobrevivencia de todos los fragmentos fue alta, variando entre 90 y $100 \%$, siendo el sobrecrecimiento algal el signo más recurrente en la mortalidad parcial. A partir de esta información, se demuestra la eficiencia a bajo costo de las guarderías flotantes como método de restauración.

Palabras clave: coral, crecimiento, sobrevivencia, estado de salud, isla San Martín de Pajarales.

\section{ABSTRACT}

Accelerated degradation of coral formations, especially shallow water species, such as $A$. cervicornis, has caused great concern, leading to the implementation of different active measures to recover this ecosystem. This study evaluated the efficiency of a vertical tree nursery model with $A$. cervicornis fragments in San Martín de Pajarales Island (Oceanario, Center for Research, Education and Recreation [CEINER], Corales del Rosario and San Bernardo National Park [PNNCR y SB, in Spanish], Colombia), where 10 vertical nurseries were installed ( 5 morfotypes). A total of 12 fragments from each morfotype were studied, determining their growth (linear and ecological volume), survival and health status. Morfotypes evaluated behaved similarly in both growths. Likewise, the survival of fragments by morfotype was high, varying from 90 to $100 \%$, with algal overgrowth being the most recurrent sign causing partial mortality. This demonstrates the low-cost efficiency of floating nurseries as a method for the restoration of this type of ecosystem.

Keywords: Coral, growth, survival, health status, San Martín de Pajarales island.

\section{INTRODUCCIÓN}

El ecosistema coralino es considerado fundamental debido a su importancia biológica y ecológica (Galvis \& Molina, 2006; Garzón-Ferreira, 1997). Infortunadamente, la cobertura de coral vivo ha disminuido como resultado de las amenazas que enfrenta este ecosistema, dando paso a un incremento de macroalgas $\mathrm{y} / \mathrm{o}$ corales no formadores de arrecifes (Moreno-Bonilla \& Valderrama, 2002; Giraldo, 2007; Murillo, 2012).

Según Alvarado, Acero y Zea (1994), el desgaste de las formaciones arrecifales en Colombia se empezó a observar con la mortalidad del género Acropora: $A$. cervicornis y A. palmata, cuya presencia es considerada fundamental en zonas de aguas poco profundas (Murillo, 2012; Zubillaga, Márquez, \& Cróquer, 2008). La disminución desmesurada de las mismas fue el resultado de la aparición de enfermedades específicas para acroporidos, como la banda blanca (Díaz et al., 1996; Sutherland, Porter, \& Torres, 2004), eventos de blanqueamiento (Aronson \& Precht, 2001) y acciones no naturales como la pesca con dinamita. Con el fin de disminuir o mitigar el daño causado se han diseñado diferentes estrategias, las cuales se clasifican en medidas activas y pasivas (Rinkevich, 2005). Las medidas activas proponen acciones como la construcción de arrecifes artificiales (Abelson \& Shlesinger, 2002; Sherman, Gilliam, \& Spieler, 2001; Van-Treeck \& Schuhmacher 1999), el trasplante de colonias de coral completas o fragmentos de estas (Garzón, 2015; Gleason, Brazeau, \& Munfus, 2001; Sanjuán, 1995; Smith \& Hughes, 1999) y el establecimiento de guarderías de coral, observando diferencias entre genotipos/ morfotipos (García, 2012; Murillo, 2012; Pizarro, Carrillo, \& García-Rueda, 2014; van Veghel \& Bosscher, 1995; Rinkevich, 1995, 2000, 2005). Por otra parte, según Ardila, Navas y Reyes (2002), se han establecido tres medidas de conservación pasiva para Colombia: la integración de las áreas coralinas en los Parques Nacionales Naturales colombianos, la ejecución del proyecto Sistema Nacional de Monitoreo de Arrecifes Coralinos en Colombia (SIMAC) y la inclusión de todos los corales dentro de los listados del Apéndice II de la Convención sobre el Comercio Internacional de Especies Amenazadas de Fauna y Flora Silvestres (CITES, en inglés).

En aras de recuperar las formaciones arrecifales del PNNCR y SB, se desarrolló un programa de restauración, monitoreo e investigación dirigido al estado ac- 
tual de $A$. cervicornis, teniendo en cuenta que la categorización en el Libro Rojo de Invertebrados Marinos de Colombia la considera en peligro crítico (PC) (Ardila et al., 2002), lo cual hace relevante la generación actualizada de información biológica y ecológica para la formulación de estrategias encaminadas a rehabilitar y manejar esta especie en áreas marinas protegidas. Por ende, el objetivo de este trabajo es evaluar el morfotipo más viable de coral $(A$. cervicornis) en términos de mayor porcentaje de crecimiento y de sobrevivencia de los fragmentos colgados en las guarderías de coral, considerando algunas de las características biológicas de la especie y las condiciones ambientales.

\section{MATERIALES Y MÉTODOS}

Cada estructura flotante consta de un ancla (varilla metálica) de $1 \mathrm{~m}$ de largo, seguida de módulos tipo árbol con una longitud de 1,54 $\mathrm{m}$, en donde se encuentran 10 varillas intercaladas de manera perpendicular ( $91,5 \mathrm{~cm}$ cada una). Cada varilla posee 10 huecos, en los cuales se sostienen los fragmentos de coral por medio de amarres de nylon, obteniendo un total de 100 fragmentos de coral por estructura. El árbol y el ancla son fijadas a partir de cuerdas verticales. El extremo superior de la estructura se une a 3 boyas con una cuerda, las cuales mantienen la estructura en posición vertical y soportan el peso de los fragmentos (figura 1). En total, se colocaron 10 estructuras flotantes (5 morfotipos), separadas entre sí por una distancia aproximada de $2 \mathrm{~m}$.

Figura 1. Esquema de guardería vertical flotante para A. cervicornis. Diseño trabajado por el CEINER

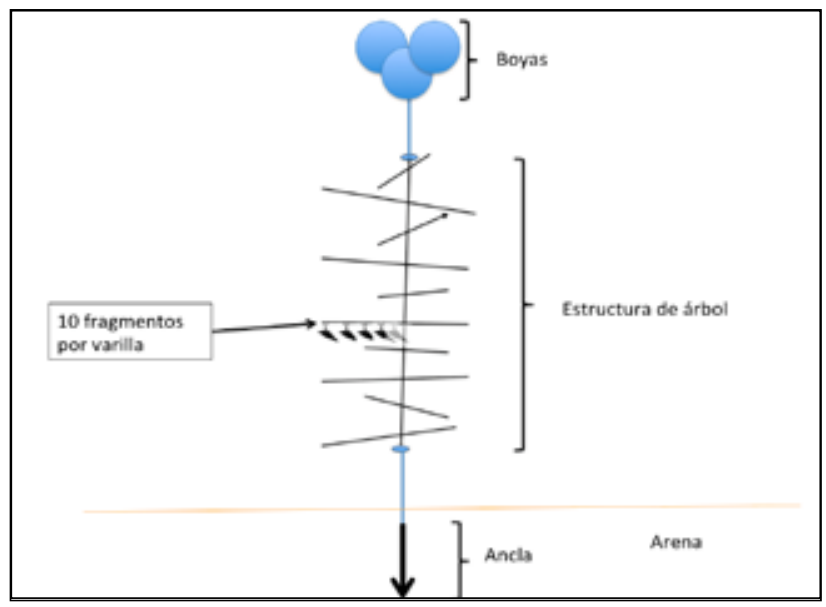

Para repoblar las 10 guarderías flotantes se tuvo en cuenta una condición de tejido adecuada, la ausencia de blanqueamiento y la ausencia de competidores (algas, esponjas, corales y/o mileporidos). Con base en las colonias sobrevivientes, se tomaron diferentes fragmentos de varios morfotipos teniendo como objetivo evaluar la sobrevivencia (\%) y el estado de salud y el crecimiento (\%) de los nuevos fragmentos. Se tuvieron en cuenta 12 fracciones por morfotipo, con un total de cinco morfotipos (O, N, K, J e I).

Con el fin de obtener medidas más precisas, los fragmentos colgados en las guarderías flotantes tenían longitudes entre $10-15 \mathrm{~cm}$, sin protuberancias. Posteriormente, las guarderías fueron monitoreadas cada 15 días por cerca de 45 días ( $\approx$ mes y medio). Durante el seguimiento, se tuvieron en cuenta 6 fragmentos por árbol (2 por morfotipo), registrando datos de sobrevivencia (muerte total o parcial o pérdida por desprendimiento), estado de salud (palidecimiento, enfermedades y sobrecrecimiento algal) y crecimiento (largo y diámetro).

La sobrevivencia (\%S) se determinó por medio de la siguiente ecuación:

$$
\% S=\frac{(\text { afragmedesprendidos }+ \text { Nfragm muertos }) x 100}{* \text { fragminiciales }}
$$

Para el análisis del estado de salud se estimó la frecuencia de ocurrencia (FO) de cada condición, calculado con la siguiente ecuación:

$$
\text { FO }=\left(\frac{\text { frag. con la condicián }}{\text { Frag. totales }}\right) \times 100 \quad \text { (Ec. 2) }
$$

Por su parte, la medición del crecimiento se realizó de dos formas: la primera comprendió la medición del crecimiento lineal (centímetros con base en el tiempo) y su diámetro (milímetros); la segunda calculó el volumen ecológico (Shafir \& Rinkevich, 2010), el cual representa el volumen de agua ocupado por los fragmentos. Al contar con las medidas de diámetro y ancho se utiliza la fórmula de volumen de un cilindro $\left(V=\pi x r^{2} h\right)$, donde $r$ es el radio $(r=(l+w) / 4$, corrección de Rinkevinch \& Loya, 1983) y $h$ la longitud del fragmento. También se determinaron las tasas exponenciales de crecimiento de las colonias con la constante de la tasa de crecimiento $(\mathrm{k})$ para los volúmenes ecológicos $(E)$, los cuales fueron 
computados mediante la fórmula de Shafir, Van-Rijin y Rinkevich (2006): $E t=E 0 * e^{k \cdot t} \quad$ siendo $k=\frac{\frac{\text { LnRt }}{t i t}}{t}$, donde $t$ corresponde al tiempo en días.

Para la fase estadística se transformaron los datos con logaritmo natural para la realización de un ANOVA, cuyo objetivo es identificar diferencias estadísticas entre los morfotipos. No se llevaron a cabo pruebas de múltiples rangos debido a que no se encontraron diferencias entre los morfotipos evaluados.

\section{RESULTADOS}

En total se colocaron aproximadamente 1.000 fragmentos de $A$. cervicornis en 10 árboles flotantes, 2 árboles por morfotipo ( $\mathrm{O}, \mathrm{N}, \mathrm{K}, \mathrm{J}$ y I). A estos se les realizó un seguimiento en cuanto a sobrevivencia y mortalidad, estado de salud y crecimiento durante 45 días ( $\approx$ mes y medio).

\section{Sobrevivencia y mortalidad}

La sobrevivencia de los fragmentos varió entre los morfotipos. Ningún fragmento presentó mortalidad por desprendimiento. Los morfotipos que evidenciaron mayores porcentajes de sobrevivencia fueron el morfotipo 0 y el morfotipo I (100 \% cada uno), seguidos por el morfotipo K $(97,916 \%)$. Los morfotipos con menores sobrevivencias fueron el morfotipo $\mathrm{N}$ $(91,666 \%)$ y el morfotipo J $(90,222 \%)$, como se muestra en la figura 2.

Figura 2. Sobrevivencia (\%) de los fragmentos de $A$. cervicornis: $\mathrm{n}=12$ (morfotipo 0 ); $\mathrm{n}=12$ (morfotipo $\mathrm{N}) ; \mathrm{n}=12$ (morfotipo $\mathrm{K}$ ); $\mathrm{n}=12$ (morfotipo J); $\mathrm{n}=12$ (morfotipo $\mathrm{I})$

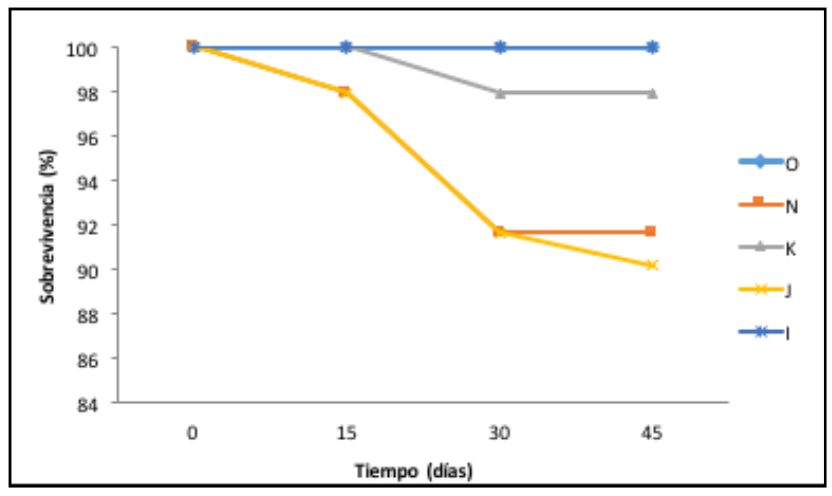

\section{Estado de salud}

Algunos de los fragmentos colgados presentaron palidecimiento y sobrecrecimiento algal. El palidecimiento en los fragmentos se empezó a observar hacia los cinco (5) primeros días de haber sido colgados (en todos los morfotipos), probablemente por el estrés sufrido al ser fragmentados. Por otra parte, el sobrecrecimiento algal se observó a los 15 días de permanencia en la estructura, afectando principalmente a los morfotipos $\mathrm{N}, \mathrm{K}$ y J hasta el último día de monitoreo (tabla 1).

Tabla 1. Estado de salud expresado como frecuencia de ocurrencia (FO) para los morfotipos de $A$. cervicornis

\begin{tabular}{|c|c|c|c|}
\hline Tiempo (días) & Morfotipo & F.0 (\%) & Signo \\
\hline 0 & 0 & 83,33 & Palidecimiento \\
0 & N & 50,00 & Palidecimiento \\
0 & J & 66,67 & Palidecimiento \\
15 & N & 16,67 & Crecimiento algal \\
15 & J & 16,67 & Crecimiento algal \\
30 & N & 16,67 & Crecimiento algal \\
30 & K & 16,67 & Crecimiento algal \\
30 & J & 16,67 & Crecimiento algal \\
45 & N & 16,67 & Crecimiento algal \\
45 & K & 16,67 & Crecimiento algal \\
45 & J & 16,67 & Crecimiento algal \\
\hline
\end{tabular}

\section{Crecimiento lineal}

Todos los fragmentos incrementaron su longitud, variando en promedio entre morfotipos. Las medidas de longitud lineal de $A$. cervicornis mostraron que no existen diferencias significativas entre los morfotipos (ANOVA, valor-P $=0,6604$ ). El mayor crecimiento de fragmentos en promedio fue de $13,342 \mathrm{~cm}$, presentado por el morfotipo $\mathrm{N}$, y el menor, en promedio, fue de 11,933 cm, exhibido por el morfotipo K (figura 3a). Por otra parte, el morfotipo que presentó mejor porcentaje de crecimiento lineal final fue el J $(11,7 \%)$ y el de menor incremento se presentó en el morfotipo $\mathrm{N}$ $(8,2 \%)$ (figura $3 b)$. 
Figura 3. a) Promedio ( \pm error estándar) del crecimiento lineal de los morfotipos de $A$. cervicornis; b) Porcentaje de crecimiento lineal de los morfotipos de A. cervicornis. $\mathrm{n}=12$ (morfotipo 0$) ; \mathrm{n}=12$ (morfotipo $\mathrm{N}) ; \mathrm{n}=12$ (morfotipo $\mathrm{K}) ; \mathrm{n}=12$ (morfotipo J); $\mathrm{n}=12$ (morfotipo I).
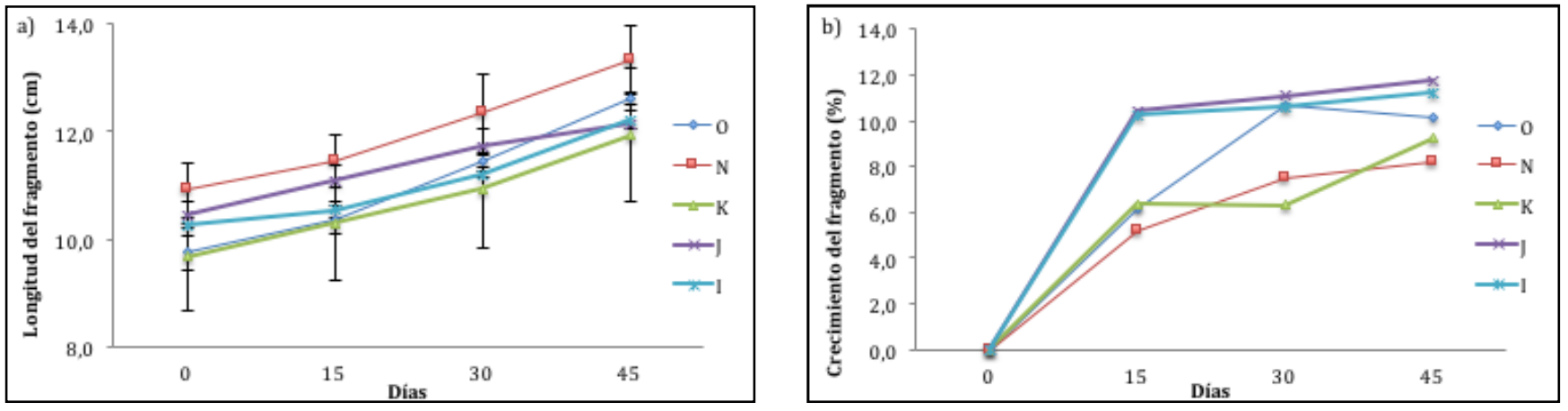

\section{Crecimiento volumen ecológico}

El mayor incremento porcentual promedio en volumen ecológico lo presentó el morfotipo O $(58,14 \%)$, seguido del morfotipo $\mathrm{N}(46,84 \%)$, como se detalla

en la tabla 2. Al respecto, los volúmenes ecológicos de A. cervicornis (figura 4) demostraron que no existen diferencias significativas entre los morfotipos (ANOVA, valor- $\mathrm{P}=0,2458)$.

Tabla 2. Volumen ecológico (VE) de los fragmentos de los morfotipos de A. cervicornis (VE inicial, VE final y VE incremento o disminución \%)

\begin{tabular}{|c|c|c|c|c|c|}
\hline Coral & Morfotipo & VE inicial $\left(\mathrm{cm}^{3}\right)$ & VE final $\left(\mathrm{cm}^{3}\right)$ & $\begin{array}{c}\text { Incremento o } \\
\text { disminución VE }\left(\mathrm{cm}^{3}\right)\end{array}$ & $\begin{array}{c}\text { Incremento o } \\
\text { disminución VE (\%) }\end{array}$ \\
\hline \multirow{3}{*}{ Acropora } & 0 & 8,02 & 12,69 & 4,66 & 58,14 \\
cervicornis & $\mathrm{N}$ & 9,14 & 13,42 & 4,28 & 46,84 \\
& $\mathrm{~K}$ & 8,18 & 10,50 & 2,32 & 28,31 \\
& $\mathrm{~J}$ & 9,37 & 11,81 & 2,43 & 25,95 \\
& $\mathrm{I}$ & 7,79 & 9,34 & 1,55 & 19,87 \\
\hline
\end{tabular}

Figura 4. a) Promedio ( \pm error estándar) del volumen ecológico de los fragmentos de $A$. cervicornis; b) Porcentaje de crecimiento (volumen ecológico) de los morfotipos de A. cervicornis: $\mathrm{n}=12$ (morfotipo O); $\mathrm{n}=12$ (morfotipo $\mathrm{N}$ ); $\mathrm{n}=12$ (morfotipo K); $\mathrm{n}=12$ (morfotipo J); $\mathrm{n}=12$ (morfotipo I)

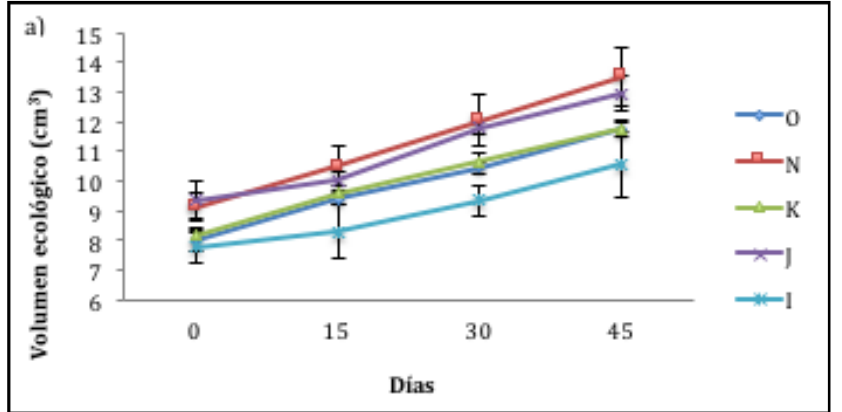

Adicionalmente, se calculó la constante de crecimiento (k) para los morfotipos de A. cervicornis, obteniendo los siguientes valores finales para los diferentes morfotipos: $1,02 \times 10^{-2}(\mathrm{O}) ; 9,5 \times 10^{-3}(\mathrm{~N}) ; 1 \times 10^{-2}(\mathrm{~K})$; $8,9 \times 10^{-3}(\mathrm{~J}) ; 1 \times 10^{-2}(\mathrm{I})$. Los resultados muestran una tendencia general de la constante de crecimiento

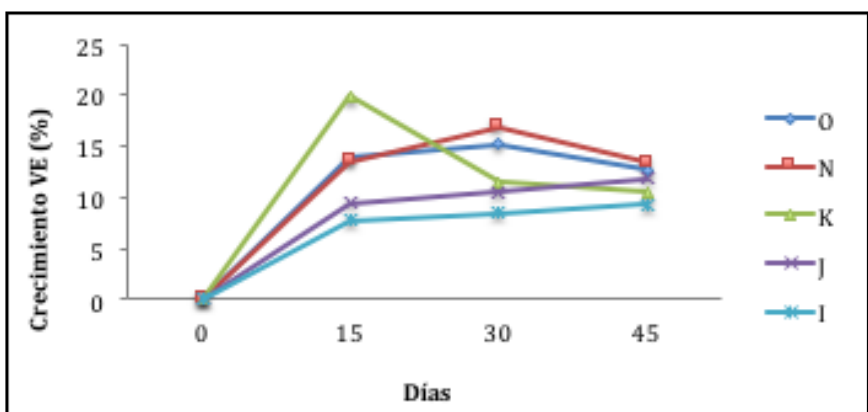

para cada morfotipo, la cual en un principio es mayor y va disminuyendo a medida que crece el coral y avanza el estudio (figura 5). 
Figura 5. Promedio de la constante de crecimiento $(k=[(1 \mathrm{n} \mathrm{Et} / \mathrm{Eo}] / \mathrm{t})$ : $\mathrm{n}=12$ (morfotipo 0 ); $\mathrm{n}=12$ (morfotipo $\mathrm{N}) ; \mathrm{n}=12$ (morfotipo $\mathrm{K}) ; \mathrm{n}=12$ (morfotipo J); $\mathrm{n}=12$ (morfotipo I)

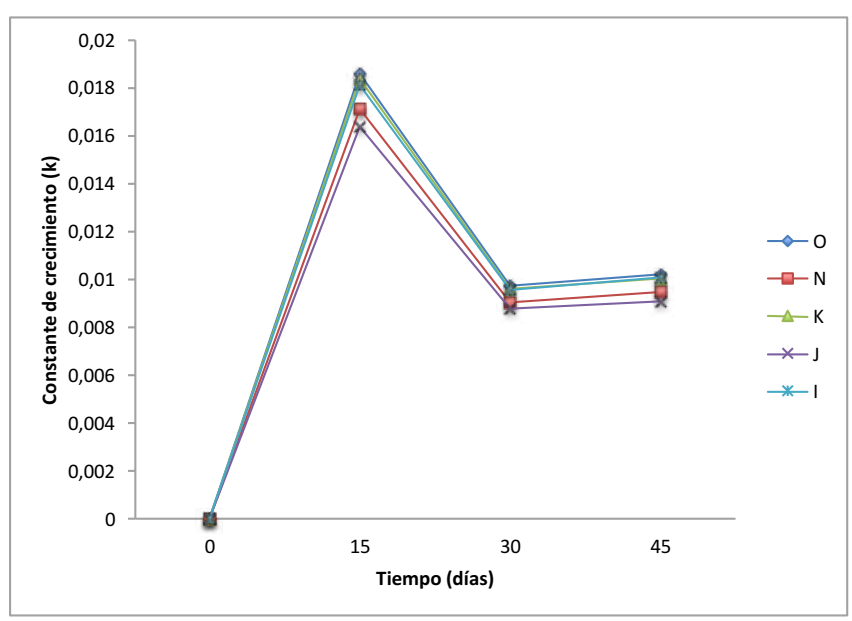

\section{DISCUSIÓN}

Los resultados de este estudio muestran altos porcentajes de sobrevivencia y crecimiento para la mayoría de los fragmentos de los 5 morfotipos evaluados. Esto se debe, principalmente, a que con el modelo de guardería flotante se reducen diferentes factores que pueden afectar a los corales, tales como la competencia, la fijación al sustrato, la depredación y/o eventos naturales como el mar de leva, el cual, según Sanjuan (1995), es uno de los factores más influyentes en la mortandad del coral cachito de venado en el PNNCR y SB debido al aumento del oleaje y los fuertes vientos.

Por otra parte, se evidenció un efecto antrópico-natural causado por el Canal del Dique, puesto que al realizar el estudio en época lluviosa menor se observó un incremento del caudal del mismo, generando cinco (5) bombazos (aumento en el caudal del Canal del Dique en época de lluvias que genera mayor carga de sedimentos) que alcanzaron a incidir en las guarderías, lo que disminuye la penetración lumínica, incrementa los nutrientes en la columna del agua y ocasiona la resuspensión de las partículas del fondo, hecho que influencia de manera directa el estado de las formaciones coralinas (Restrepo-Ángel \& Alvarado, 2011) y de los fragmentos de las guarderías flotantes.

\section{Sobrevivencia y mortalidad}

Ciertos factores incidieron en la sobrevivencia y la mortalidad parcial de los fragmentos para algunos morfotipos a los 15 días y/o al mes de haber sido colocados en las estructuras. Esto debido a que, según Shafir et al. (2006), el primer mes de guardería es un periodo crítico, puesto que algunos de los segmentos de coral sufren demasiado estrés y pueden ser colonizados por organismos oportunistas.

Durante el mes y medio de estudio no se evidenció mortalidad total para ninguno de los morfotipos, lo cual ocurrió, probablemente, por la buena calidad del agua, el bajo oleaje para la época, el tiempo de estudio y la rápida respuesta del coral para adherirse al nylon. En general, contrario a lo reportado por $\mathrm{Mu}$ rillo (2012) y García (2012), no se presentaron pérdidas por desprendimiento de fragmentos, gracias a que para el presente trabajo estos poseían una longitud mayor (10-15 cm), en comparación con los experimentos de estos autores, evitando o disminuyendo la probabilidad de mortalidad por pérdida del fragmento. Sin embargo, existieron otras fuentes que pudieron debilitar los fragmentos, como la incidencia del Canal del Dique. A pesar de ello, no se evidenció un cambio drástico en los factores fisicoquímicos del agua, para la cual se reportaron temperaturas entre $28-30$ 으, superando en un grado el rango óptimo, según CONABIO (2011), una salinidad promedio de 33,9 y valores de oxígeno disuelto entre 6 y $9 \mathrm{mg} / \mathrm{l}$; parámetros adecuados para el crecimiento de especies como $A$. cervicornis que determinan el éxito del cultivo (Gattuso et al., 1998; Herlan \& Lirman, 2008).

Es importante recalcar que, probablemente, el éxito en la sobrevivencia de los fragmentos de las estructuras flotantes ubicadas en la isla San Martín de Pajarales se deba a factores como el tiempo de estudio, la baja influencia turística (buceo y paso de lanchas) y la profundidad a la que se trabajó (no mayor a $5 \mathrm{~m}$ ). Esto concuerda con los resultados de Murillo (2012) para la especie "cachito de venado", la cual mostró mejores tasas de crecimiento y sobrevivencia a menores profundidades.

\section{Estado de salud}

Las principales afecciones o signos de deterioro observados en los fragmentos de coral $A$. cervicornis fueron el palidecimiento y el sobrecrecimiento de algas. El palidecimiento de los fragmentos de coral sin causar mortalidad solo se apreció entre los 0 y 15 días, lo cual indicó un estrés intenso en los segmentos utili- 
zados como resultado, posiblemente, del manejo de la colonia donadora y la fragmentación de la misma; liberando por medio del moco algunas de las zooxantelas y generando una coloración no tan viva del coral. Algunos corales como el "cachito de venado" pueden retener hasta el $10 \%$ de las algas simbiontes y restablecerlas al terminar las condiciones de estrés (Fitt, McFarland, Warner, \& Chilcoat, 2000).

Según Murillo (2012), existen otros factores que intervienen en el palidecimiento de estas especies, entre los que se encuentran la temperatura, la sedimentación y la irradiación. Esta última se evidenció al inicio del proyecto cuando se identificaron fragmentos con una mitad pálida (hacia la luz solar) y otra mitad normal (contraluz), lo cual pudo haber ocurrido por fotoinhibición del simbionte como consecuencia de las bajas profundidades o condiciones de estrés oxidativo. Posteriormente, se observó que las algas simbiontes de las áreas saludables se traslocaron hacia las zonas pálidas, devolviendo la coloración normal a todo el fragmento. Según Warner, Fitt y Schmidt (1999), este palidecimiento se debe a la reducción de pigmentos fotosintéticos del alga (Symbiodinium sp) y no a la expulsión de la misma. Dicha reducción puede ocasionarse como una defensa que disminuye el daño en el fotosistema II del alga.

Por otra parte, el sobrecrecimiento algal es un factor que afecta a las poblaciones de Acropora cervicornis en el PNNCR y SB permanentemente (García, 2012; Murillo, 2012; SanJuan, 1995). Sin embargo, este problema no hace referencia solo a esta zona, sino que también ha sido reportado en el Parque Nacional Natural Tayrona (PNNT) y en la región Caribe del país (Galvis \& Molina, 2006; Moreno-Bonilla \& Valderrama, 2002). En este trabajo no se apreció gran incidencia de algas sobre los corales debido al bajo tiempo de seguimiento de los fragmentos colgados. Las algas analizadas en este estudio solo afectaron parcialmente al coral y puede que su incidencia haya sido facilitada por un bombazo del Canal del Dique.

Otro vector de algas puede ser la presencia de la "damisela" Stegastes planifrons, especie que cultiva céspedes, según Santodomingo et al. (2002), contribuyendo de manera importante a la pérdida de tejido coralino y desempeñando un papel significativo en la dinámica de las formaciones arrecifales. En este trabajo no se observaron damiselas en los fragmentos, pero es posible que posteriormente sean colonizados por estas a medida que el fragmento toma tridimensionalidad, como se observó en las colonias donantes. El sobrecrecimiento algal fue relativamente bajo debido, posiblemente, al diseño de estructura flotante, el cual no brinda sustrato para las algas.

\section{Crecimiento}

Como se observó en los resultados, la tasa de crecimiento $(\mathrm{k})$ de los fragmentos siguió una tendencia de disminución a medida que pasaba el tiempo (días), lo que concuerda con los hallazgos de Padilla y Lara (1996), quienes encontraron que la tasa de crecimiento de las colonias jóvenes (recién fragmentadas) es mayor con el fin de incrementar su probabilidad de sobrevivencia, constante que disminuye a medida que pasa el tiempo. Esto mismo se corrobora en los trabajos de Murillo (2012) y García (2012).

Algunos de los factores que inciden en el crecimiento de los fragmentos son la temperatura y la intensidad de la radiación ultravioleta (UVR), lo que explicaría los resultados favorables del estudio. La temperatura identificada en el presente estudio se encuentra entre los rangos óptimos en los que la especie puede sobrevivir, crecer y reproducirse. Por otro lado, el crecimiento también pudo haber sido afectado por la intensidad UVR. Según Torres, Armstrong, Corredor y Gilbes (2007), los cambios en la intensidad UVR producen una disminución en la concentración de pigmentos fotosensibles y en el crecimiento. Este estudio no tuvo en cuenta la intensidad UVR, por lo cual es necesario que futuros trabajos lleven a cabo la evaluación de esta variable teniendo en cuenta la sobrevivencia y el crecimiento entre diferentes morfotipos de $A$. cervicornis, puesto que, según Rinkevich (2000), estas variables no solo dependen de cambios ambientales, sino de caracteres genéticos; por ello, se puede afirmar que los fragmentos crecieron de manera adecuada en las guarderías durante este periodo crítico. Al comparar con los resultados de Sanjuan (1995), se constata la buena tasa de crecimiento del coral $A$. cervicornis para el PNNCR y SB. Por el contrario, si se confronta con el trabajo de Murillo (2012) para PNNT, las tasas de crecimiento son mucho más bajas allí.

Comparando a fondo los valores obtenidos con los resultados de Murillo (2012) para A. cervicornis (mayor volumen ecológico con $2,5 \mathrm{~cm}^{3}$ y $2,6 \mathrm{~cm}^{3}$ ), la utilización de las guarderías flotantes en el PNNCR y SB es 
más eficiente que en el PNNT, ya que se observaron mejores porcentajes de crecimiento al mes y medio de colgados los fragmentos. Por otra parte, también es necesario aclarar que las condiciones ambientales de las zonas varían principalmente por la duración de diferentes efluentes, como el Manzanares y Gaira (PNNT), mientras que los bombazos del Canal del Dique (PNNCR y SB) no son permanentes, con una duración en la zona de las guarderías, según lo observado, de menos de un día. Sin embargo, estos datos son los únicos comparables publicados para $A$. cervicornis en el Caribe colombiano, ya que aunque existen trabajos más actualizados sobre la especie, como el de Zarza et al. (2014), se continúan trabajando medidas de crecimiento lineal. Por tal motivo, no es posible realizar comparaciones contundentes entre los estudios desarrollados.

No se encontraron diferencias significativas entre los fragmentos de varios morfotipos del coral "cachito de venado" (ni para crecimiento lineal, ni para volumen ecológico), hecho que probablemente se deba al tiempo de muestreo. No obstante, descriptivamente se pudo observar un buen crecimiento de los morfotipos J e I, lo cual puede estar relacionado con lo expresado por Rinkevich (2000), quien sostuvo que el crecimiento no depende únicamente de cambios en el ambiente, sino también de características genéticas. Por ende, es importante realizar estudios con un mayor tiempo de duración para comprobar si existen diferencias o no en el crecimiento de los genotipos/morfotipos de A. cervicornis, como lo comprobaron van-Veghel y Bosscher (1995) para Orbicella annularis, quienes demostraron bajo las mismas condiciones ambientales que existieron diferentes tasas de crecimiento del coral modular a lo largo de 3 años de muestreo.

A pesar de la poca información existente para el Caribe colombiano (Murillo, 2012; García, 2012) sobre la medición de volumen ecológico, este es uno de los métodos más utilizados para determinar el crecimiento tridimensional del fragmento en guardería (Shafir et al., 2006). Al ser fragmentados, los corales, en cierta fase, reducen su tasa de crecimiento vertical e incrementan su extensión lateral, lo cual aumenta el volumen de agua ocupado por los fragmentos (Shaish et al., 2010). Lo anterior se puede constatar en los resultados obtenidos en la tercera medición del estudio (mes), obteniendo un efecto importante sobre la nutrición heterotrófica del coral (Bruno \& Edmunds, 1998).

Por último, la expectativa en cuanto a la restauración coralina es buena, debido a que de pequeños fragmentos se generan grandes colonias, tal como reportan algunos trabajos (SanJuan, 1995; Shafir et al., 2006). Sin embargo, es de suma importancia llevar un monitoreo continuo, ya que las condiciones ambientales no son siempre óptimas y varían continuamente.

\section{CONCLUSIONES Y RECOMENDACIONES}

Con base en los resultados obtenidos, se infiere que el uso de guarderías flotantes es un método viable para el desarrollo de coral A. cervicornis en el PNNCR y SB, incrementando de manera significativa la sobrevivencia y el crecimiento del mismo y reduciendo la mortalidad ocasionada por la competencia por espacio y la depredación y/o colonización de organismos epibiontes. A pesar de esto, las estructuras flotantes son solo una fase inicial del programa de restauración de corales, lo que conlleva a procesos más complejos en el medio natural.

Debido a que es necesario conocer el crecimiento del fragmento (no solo de forma lineal, sino tridimensional), el método más completo para llevar a cabo este análisis es el volumen ecológico, puesto que ofrece mayor información sobre las colonias. Por lo tanto, se recomienda la utilización de este método en trabajos posteriores sobre el tema.

No se encontraron diferencias significativas entre los morfotipos del coral "cachito de venado", por lo que resulta pertinente considerar un mayor lapso de tiempo para este tipo de trabajos a fin de llegar a conclusiones más fehacientes sobre cuál de los morfotipos podría ser el más apto para ser trasplantado en el medio natural. Se sugiere realizar estudios moleculares para observar qué secuencias genéticas son las que cambian entre los morfotipos y puedan estar afectando o mejorando su sobrevivencia.

El éxito de la sobrevivencia de la cría de fragmentos en guarderías radica principalmente en el fácil manejo, control y seguimiento que pueda hacerse a estos. De igual manera, es importante llevar a cabo jornadas de limpieza para evitar la competencia con algas, corales no formadores de arrecife y organismos bioero- 
dadores, optimizando así los valores de sobrevivencia y crecimiento de los fragmentos.

Se propone continuar evaluando la respuesta de $A$. cervicornis en las diferentes épocas del año, puesto que el seguimiento por un periodo mayor podría mostrar cambios de sobrevivencia en los morfotipos evaluados, así como diferentes tendencias de crecimiento en función de las condiciones ambientales.

\section{REFERENCIAS}

Abelson, A., \& Shlesinger, Y. (2002). Comparison of the development of coral and fish communities on rock-aggregated artificial reefs in Eilat, Red Sea. ICES Journal of Marine Science, 59, 122-126.

Alvarado, E., Acero, A., \& Zea, S. (1994). El ecosistema arrecifal de Colombia. En CCO, ENB, y Colciencias. Memorias del taller de expertos sobre el estado del conocimiento y lineamientos para una estrategia nacional de biodiversidad en los sistemas marinos y costeros: recopilación de los documentos básicos informativos sobre la biodiversidad costera y marina en Colombia. Magdalena, Colombia, pp. $150-175$.

Ardila, N., Navas, G., \& J. Reyes. (2002). Libro rojo de invertebrados marinos de Colombia. La serie libros rojos de especies amenazadas de Colombia. Bogotá: INVEMAR/Ministerio de Medio Ambiente.

Aronson, R., \& Precht, W. (2001). White-band disease and the changing face of Caribbean coral reefs. Hydrobiologia, 460, 25-38.

Bruno, J. F., \& Edmunds, P. J. (1998). Metabolic consequences of phenotypic plasticity in the coral Madracis mirabilis (Duchassaing and Michelotti): the effect of morphology and water flow on aggregate respiration. Journal of Experimental Marine Biology and Ecology, 229, 187-195.

Comisión Nacional para el Conocimiento y Uso de la Biodiversidad [CONABIO] (2011). Fichas de especies prioritarias. Coral Cuerno de Alce y Coral Cuerno de Ciervo. México D. F.: ConABIo.

Díaz, J. M., Díaz, G., Garzón-Ferreira, J., Geister, J., Sánchez, J., \& Zea, S. (1996). Atlas de los Arrecifes
Coralinos del Caribe Colombiano. Complejos arrecifales. Santa Marta: INVEMAR.

Fitt, W. K., McFarland, F. K., Warner, M., \& Chilcoat, G. C. (2000). Seasonal patterns of tissue biomass and densities of symbiotic dinoflagellates in reef corals and relation to coral bleaching. Limnology and Oceanography, 45, 677-685.

Galvis, I., \& Molina, B. (2006). Sobrevivencia, crecimiento, salud y reclutamiento de Acropora palmata (Lamark, 1816) y Acropora cervicornis (Lamark, 1816) en el Parque Nacional Natural Tayrona (PNNT) y en el Parque Nacional Natural Corales del Rosario y San Bernardo (PNNCRSB). Tesis. Universidad Jorge Tadeo Lozano, Colombia. 85p.

García, A. (2012). Cría de fragmentos de Acropora palmata y Montastrea cavernosa en una guardería a media agua en la Bahía de Gayraca (Parque Nacional Natural Tayrona) como aproximación hacia la restauración coralina. Trabajo de grado. Universidad Jorge Tadeo Lozano. Colombia.

Garzón-Ferreira, J. (1997). Arrecifes Coralinos: ¿Un tesoro camino a la extinción? Ciencia y Tecnología, 15(1), 11-19.

Garzón, M. (2015). Evaluación de crecimiento y supervivencia de fragmentos de Acropora cervicornis trasplantados al medio natural, provenientes de cultivo en guarderías a media agua en el Parque Nacional Natural Corales del Rosario y San Bernardo. Trabajo de Grado. Universidad de Bogotá Jorge Tadeo Lozano Estado, Colombia.

Gattuso, J. P., Frankignoulle, M., Bourge, I., Romaine, S., \& Buddemeier, R. W. (1998). Effect of calcium carbonate saturation of seawater on coral calcification. Global and Planetary Change, 18(12), 37-46.

Giraldo, A. (2007). Estado actual de los corales Acropora cervicornis Lamarck, 1816) y Acropora palmata (Lamark, 1816) y Diploria Labyrinthiformis (Linnaeaus, 1758) en el Parque Nacional Natural Corles del Rosario y San Bernardo. Tesis de pregrado. Universidad de Bogotá Jorge Tadeo Lozano, Colombia. 
Gleason, D., Brazeau, D., \& Munfus, D. (2001). Can self-fertilizing coral species be used to enhance restoration of Caribbean reefs? Bulletin of Marine Science, 69(2), 933-943.

Herlan, J., \& Lirman, D. (2008). Development of a coral nursery program for the threatened coral Acropora cervicornis in Florida. In Proceedings $11^{\text {th }}$ International Coral Reef Symposium. Fort. Lauderdale, Florida.

Moreno-Bonilla, M., \& Valderrama, J. (2002). Estado actual y crecimiento de las especies coralinas Acropora pamata (Lamarck) Veron, 2000 y Acropora cervicornis (Lamarck) Veron, 2000 en el Parque Nacional Natural Tayrona, Caribe Colombiano. Tesis de pregrado. Universidad de Bogotá Jorge Tadeo Lozano, Colombia.

Murillo, I. (2012). Experimentos iniciales para la restauración de las especies Acropora cervicornis y A. palmata en el área arrecifal de Playa del Muerto (Parque Nacional Natural Tayrona). Trabajo de grado. Universidad de Bogotá Jorge Tadeo Lozano, Colombia.

Padilla, C., \& Lara, M. (1996). Efecto del tamaño de las colonias en el crecimiento de Acropora palmata en Puerto Morelos, Quinta Roo, México. Hidrobiológica, 6(6), 17-24.

Pizarro, V., Carrillo, V., \& García-Rueda, A. (2014). Revisión y estado del arte de la restauración ecológica de arrecifes coralinos. Biota Colombiana, 15(S2), 132-149.

Restrepo-Ángel, J. D., \& Alvarado, E. M. (2011). Los sedimentos del río Magdalena y su impacto sobre los arrecifes coralinos de las Islas del Rosario: una prioridad de investigación nacional. Pp. 78-94. En Zarza, E. El entorno ambiental del Parque Nacional Natural Corales del Rosario y de San Bernardo (pp. 78-94). Cartagena de Indias, Colombia: Parques Nacionales Naturales del Colombia, Banco Mundial, Global Environment Facility (GEF) \& Patrimonio Natural.

Rinkevich B., \& Loya, Y. (1983). Short term fate photosynthetic products in a hermatypic coral. Journal of Experimental Marine Biology and Ecology, 73, 175-184.
Rinkevich, B. (1995). Restoration strategies for coral reefs damaged by recreational activities: the use of sexual and asexual recruits. Restoration Ecology, $4,241-251$.

Rinkevich, B. (2000). Steps towards the evaluation of coral reef restoration by using a small branch fragments. Marine Biology, 136, 807-812.

Rinkevich, B. (2005). Conservation of Coral Reefs through Active Restoration Measures: Recent Approaches and Last Decade Progress. Environmental Science \& Technology, 39, 43334342.

Santodomingo, N., Rodríguez-Ramírez, A., \& GarzónFerreira, J. (2002). Territorios del pez Stegastes planifrons en formaciones coralinas del Parque Nacional Natural Tayrona, Caribe colombiano: un panorama general. Boletín de Investigaciones Marinas y Costeras, 31, 65-84.

Sanjuan, A. (1995). Crecimiento, sobrevivencia, reproducción y regeneración de la especie coralina Acropora cervicornis en el Parque Natural Nacional Corales del Rosario. Tesis de pregrado. Universidad de Bogotá Jorge Tadeo Lozano, Colombia.

Shaish, L., Levy, G., Katzir, G., \& Rinkevich, B. (2010). Employing a highly fragmented, weedy coral species in reef restoration. Ecological Engineering, 36(10),1424-1432.

Shafir, S., Van-Rijin, J., \& Rinkevich B. (2006). Steps in the construction of underwater coral nursery, an essential component in reef restoration acts. Marine Biology, 149, 679-687.

Shafir, S., \& Rinkevich, B. (2010). Integrated longterm mid-water coral nurseries: A management instrument evolving into a floating ecosystem. University of Mauritius Research Journal, 16, 365386.

Sherman, R. L., Gilliam, D. S., \& Spieler, R. E. (2001). Site-dependent differences in artificial reef function: implications for coral resto- ration. Bulletin of Marine Science, 69(2), 1053-1056.

Smith, L., \& Hughes, T. (1999). An experimental assessment of survival re-attachment and fecundity 
of coral fragments. Journal of Experimental Marine Biology and Ecology, 1(1), 147-164.

Sutherland, K. P., Porter, J. W., \& Torres, C. (2004). Disease and immunity in Caribbean and IndoPacific zooxanthellate corals. Marine Ecology Progress Series, 266, 273-302.

Torres, J. A., Armstrong, R. A., Corredor, J. E., \& Gilbes F. (2007). Physiological responses of Acropora cervicornis to increased solar irradiance. Photochemistry and Photobiology, 83, 839-850.

Van-Veghel, M. L. J., \& Bosscher, H. (1995). Variations in linear growth and skeletal density within the polymorphic reef building coral Montastrea annularis. Bulletin of Marine Science, 56(3), 902908.

Van-Treeck, P., \& Schumacher H. (1999). Artificial reefs created by electrolysis and coral transplantation: an approach ensuring the compatibility of environmental protection and diving tourism. Estuarine, Coastal and Shelf Science, 49(S1), 75-81.

Warner, M. E., Fitt, W. K., \& Schmidt, W. (1999). Damage to photosystem II in symbiotic dinoflagellates: a determinant of coral bleaching. Proceedings of the National Academy of Sciences, 96(14), 8007-8012.

Zarza, E., Vargas, A., Londoño, L., Pacheco, A., \& Duque, D. (2014). Ensayo preliminar de crecimiento de fragmentos del coral amenazado Acropora cervicornis en una guardería colgante y experiencia piloto de trasplante en el Parque Nacional Natural Corales del Rosario y de San Bernardo, Caribe colombiano. Biota Colombiana, 15(S2), 102-113.

Zubillaga, A., Márquez L., \& Cróquer A. (2008). Ecological and genetic data indicate recovery of the endangered coral Acropora palmata in Los Roques, Southern Caribbean. Coral Reefs, 27(1),63-72. 